\title{
Antimicrobial and antioxidant effects of phlorotannin coating in spoilage prevention of tomato during storage
}

\begin{abstract}
The microbial flora present on vegetables and fruit is commercially significant as they cause spoilage and potential health risks in consumers. Synthetic chemicals used to combat food microbes posed serious health and environmental hazards leading to the search for natural alternatives. In this study, phlorotannins from three Indian brown seaweeds, Sargassum myriocystum, Turbinariaornata, and Padina tetrastromatica were evaluated for antimicrobial, antioxidant, and fruit spoilage prevention potentials. The crude phlorotannin of Padina with the highest content $\left(0.114 \pm 0.0005 \mathrm{~g} \mathrm{~kg}^{-1}\right)$ and antioxidant activity $(92.65 \pm 3.43 \%)$ was purified by column chromatography. The Padina water fraction I with the highest antioxidant potential was characterized by Fourier Transform Infra-Red spectroscopy and a $5 \%$ solution was applied as a coating on tomatoes segregated into control chilled (CC), treated chilled (TC), control non-chilled (CNC) and treated non chilled (TNC). Spoilage parameters were studied over twenty days, using a swab and spread plate methods to assess the microbial load on tomatoes. Compared to the control sets, the treated sets exhibited a low number of microbial colonies and retained a fresh look and yellow color. The antibacterial and antioxidant properties of phlorotannin coating as a natural alternative for preservatives, effectively reduced bacterial load, inhibiting the early decay of tomatoes.
\end{abstract}

Keywords: antimicrobial, phlorotannins, antioxidant, padinatetrastromatica, food spoilage, postharvest storage
Volume 7 Issue 4 - 2020

\author{
Sreekala Kannikulathel Gopidas, Nagaraj \\ Subramani \\ Centre for Advanced Studies in Botany, University of Madras, \\ India
}
Correspondence: Nagaraj Subramani, Assistant Professor, Centre for Advanced Studies in Botany, University of Madras, Guindy Campus, Chennai, Tamil Nadu, India, 600025 , Tel 044-22202760/+9I 999420I522, Email nagailly@gmail.com

Received: August 16, 2020 | Published: August 31, 2020
Abbreviations: $\mathrm{CC}$, control chilled; TC, treated chilled; $\mathrm{CNC}$, control non-chilled; TNC; treated non-chilled; TPC, total phenolic content; WF, water fraction, MF, methanol fraction; UV, ultraviolet; GA, gallic acid; DPPH, 2, 2-diphenyl-1-picrylhydrazyl; SD, standard deviation; FTIR, fourier transform infrared; $\mathrm{KBr}$, potassium bromide; CFU, colony forming units; DMSO, dimethyl sulfoxide; AP, aqueous phase; GAE, gallic acid equivalent

\section{Introduction}

The surface of vegetables and fruit harbor a variety of microbes including bacteria and fungi. The diversity and population of these species depend on several factors such as the farming environment, type of plant cultivars, postharvest handling, transportation, and storage conditions. ${ }^{1}$ These microbes slowly degrade the crop causing early spoilage during storage. They may be pathogenic or nonpathogenic, and some are even resistant to antibiotics. On consuming raw vegetables and fruit, these organisms enter the human gut leading to disease outbreaks and allergies. ${ }^{2}$ Many techniques are available for the spoilage prevention of easily perishable commodities. The use of antimicrobial compounds, fungicides, and synthetic chemical preservatives is a common practice. These preservatives compromise the organoleptic characteristics and nutritional quality of the crop. ${ }^{3}$ In the course of time, such chemicals caused serious health and environmental impacts which stirred up the research community to discover natural alternatives for the preservation of easily spoiled vegetables and fruit.

Brown seaweeds are treasure houses of numerous commercially significant compounds employed in diverse fields. Phlorotannins are phenolic compounds exclusive to brown algae, and form the largest group of secondary metabolites identified in algae. ${ }^{4}$ They are polymers of phloroglucinol units formed in the acetate-malonate pathway, as a part of the defense mechanism in algae and are broadly classified into three as fucols, phlorethols and fuhalols, and eckols and carmalols ${ }^{5,6}$ and the molecular weight ranges from $126-6500 \mathrm{Da}^{7}{ }^{7}$ Several biological activities have been reported for phlorotannins, viz, antioxidant, antiinflammatory, antimicrobial, antiprotozoal, antifungal, antimalarial, anticancer, ${ }^{8}$ antidiabetic, ${ }^{9}$ and anti-arthritic. ${ }^{10,11}$ Hence they are used as ingredients in drugs, animal feeds, diet supplements, fertilizers, nutraceuticals, and cosmetics. ${ }^{12}$

There are numerous reports on the antimicrobial and antioxidant activities of methanolic extracts and phlorotannins from brown algae. ${ }^{13,14}$ Phlorotannins purified from the various Phaeophyceaen members like E. bicyclis, E. kurome, H. fusiformis, and $E$. cavahave exhibited superior antioxidant properties with 2-10 folds increased free radical scavenging activity than catechin, $\alpha$-tocopherol and ascorbic acid. ${ }^{15}$ The methanol extracts of Sargassum wightiiand Turbinariaornatahave polyphenols responsible for their antioxidant and antibacterial activities. ${ }^{16}$ The phlorotanninrich extracts of Cystoseiranodicaulis, Cystoseiratamariscifolia, and Cystoseirausneoideswere found to possess antibacterial and antifungal properties. ${ }^{17}$ The bacteriostatic and bactericidal effects of phlorotannins from Ascophyllum nodosum against rumen microbes in cattle and of phlorotannins from Turbinariaconoides and Padina gymnospora on E. coli has been reported. ${ }^{4}$ On the other hand, an attempt to use phlorotannin as a coating on fruit or vegetables to protect them from decay has not yet been undertaken. In this context, the present study aimed to extract phlorotannins from the three Indian 
brown seaweeds, Sargassum myriocystum, Turbinariaornata, and Padina tetrastromatica, use it as a coating on tomatoes and to evaluate its antimicrobial and antioxidant activities to assess the spoilage prevention potential.

\section{Materials and methods}

\section{Materials}

The chemicals and analytical grade reagents were purchased from Hi-Media and Sisco Research Laboratories, Mumbai and Chennai, India.

\section{Collection of seaweeds}

Fresh, healthy specimens of the seaweeds were collected from the Kilakarai coast (latitude $9^{\circ} 14^{\prime} \mathrm{N}$ and longitude $78^{\circ} 50^{\prime} \mathrm{E}$ ), Gulf of Mannar located in the Southeast coast of Tamil Nadu, India. The algae were identified by experts in the Centre for Advanced Studies in Botany, University of Madras, Tamil Nadu, India. After washing thoroughly in tap water to remove all epiphytes, sand particles, and associated fauna, the seaweeds were shade dried for five days, followed by oven drying for $12 \mathrm{~h}$ at $60^{\circ} \mathrm{C}$. The material was ground to a coarse powder using mixer-grinder (Philips HL 1643/04 Vertical Mixer Grinder, India).

\section{Preparation of seaweed extracts}

Twenty grams of algal powder was extracted with $250 \mathrm{~mL}$ of $80 \%$ methanol, stirred on an incubator shaker (Lark Innovative Fine Teknowledge, Chennai, India) at $28^{\circ} \mathrm{C}$ for $24 \mathrm{~h}$. The extract was filtered and the process was repeated thrice using $100 \mathrm{~mL}$ of fresh $80 \%$ methanol. The combined extracts were filtered using a funnel and Whatman filter paper No.1 and concentrated using a rotary evaporator (Lark Innovative Fine Teknowledge, Chennai, India) operated at $\leq 35^{\circ} \mathrm{C}$, to evaporate off the methanol. The total phenolic content (TPC) and the antioxidant activity of the resulting aqueous solution were determined. The aqueous filtrate of Padina that showed the maximum content and activity was transferred into a clean separating funnel and partitioned by adding one volume of dichloromethane. The solution was vigorously shaken and left to stand for $10 \mathrm{~min}$ for phase separation. The bottom dichloromethane layer containing lipids was discarded and the partitioning procedure was repeated thrice using fresh $20 \mathrm{~mL}$ dichloromethane. The partitioned aqueous layer was collected into a pre-weighed $20 \mathrm{~mL}$ scintillation vial and evaporated residual dichloromethane using a rotary evaporator operated at $\leq 35^{\circ} \mathrm{C}$, to obtain a polyphenolic extract which was stored at $-20^{\circ} \mathrm{C}$.

\section{Preparation of phlorotannin-enriched fraction}

Silica gel (60-120 mesh size; Sisco Research Laboratories, Chennai, India) column chromatography was carried out with the above extract to obtain phlorotannin-enriched fractions. A column chromatography tube loaded with silica gel slurry was preconditioned with $12 \mathrm{~mL}$ methanol followed by $18 \mathrm{~mL}$ deionized water. The extract was dissolved in $10 \mathrm{~mL}$ deionized water and sonicated for $10 \mathrm{~min}$ for proper dissolution. This sample was loaded onto the preconditioned column cartridge using a glass Pasteur pipette. The column cartridge was eluted with $20 \mathrm{~mL}$ deionized water. The resulting eluant was collected as $10 \mathrm{~mL}$ each into two glass vials (water fraction I \& II or WF I \& WF II). Then the column cartridge was eluted with 30 $\mathrm{mL}$ methanol and collected the eluant as $10 \mathrm{~mL}$ each into three vials (methanol fraction I, II \& III or MF I, MF II \& MF III). The water fractions and methanol fractions were concentrated on a rotary evaporator operated at $\leq 35^{\circ} \mathrm{C}$, lyophilized (Mini-lyodel, Delvac Pumps Pvt. Ltd., Chennai, India), sealed in glass vials and stored at $-20^{\circ} \mathrm{C}$.

\section{Estimation of total phenolic content (TPC)}

The concentration of phenolics in algal extracts, water fractions, and methanol fractions were determined using spectrophotometric method. ${ }^{18}$ The absorbance was determined using a UV-visible spectrophotometer (Shimadzu, UV-1700, Japan) at $\lambda_{\max }=765 \mathrm{~nm}$. The samples were prepared in triplicate and the mean value of absorbance was obtained. The standard used was Gallic acid. The phenolic content was expressed in terms of Gallic acid equivalent ( $\mathrm{g}$ of GA per $\mathrm{kg}$ of extract).

\section{In-vitroantioxidant activity}

DPPH (2, 2-diphenyl-1-picrylhydrazyl) radical scavenging assay. The DPPH radical scavenging assay was carried out. ${ }^{19} 100 \mu \mathrm{L}$ each of algal extracts, water, and methanol fractions were tested. Absorbance was taken at $516 \mathrm{~nm}$ in spectrophotometer after $15 \mathrm{~min}$, using methanol as blank. The free radical scavenging activity was calculated as:

$\%$ Antiradical activity $=\frac{\text { Control absorbance }- \text { Sample absorbance }}{\text { Control absorbance }} * 100$

Each experiment was carried out in triplicate, and results are expressed as mean $\%$ antiradical activity $\pm \mathrm{SD}$.

\section{Reducing power assay}

Briefly, the reducing power of the extracts was evaluated..$^{20}$ The absorbance of all samples was measured at $700 \mathrm{~nm}$. L-ascorbic acid was used as a standard. The $\%$ of reducing power was calculated by the same equation above.

\section{Fourier transform infrared (FT-IR) spectroscopy}

The WF I that exhibited the highest total phenolic content and the highest antioxidant activity was characterized by Perkin Elmer System One (PerkinElmer Pvt. Ltd., Maharashtra, India) FTIR between $450-4000 \mathrm{~cm}-1$, at 64 scans, $4 \mathrm{~cm}-1$ wavenumber, and resolution transmission mode under standard environmental conditions. Sample ( $2 \mathrm{mg}$ ) was ground evenly with approximately $100 \mathrm{mg} \mathrm{KBr}$ until particles measured $<2.5 \mathrm{~mm}$ in size. The FTIR spectra were obtained and the signals were compared to a background spectrum collected from the $\mathrm{KBr}$ alone at room temperature.

Assessment of antimicrobial activity and spoilage prevention potential of phlorotannin (WF I) on tomato (Modified method of de Corat et al. ${ }^{21}$ The assessment of antimicrobial activity and spoilage prevention potential of Padina phlorotannin (WF I) on tomato was carried out by the modified method of de Corato et al. ${ }^{21}$

\section{Sample collection and processing}

The fresh tomatoes selected for antimicrobial and shelf-life studies, procured from a local farm in Chennai, Tamil Nadu, India, were collected in sterile polythene ziplock bags to avoid handling contamination and transported to the laboratory. Replicate samples were collected from discrete fruit. The sample was ensured to be not stocked. Since tomatoes are intrinsically fragile, prone to physical damage and have a limited shelf life, it was taken care of to obtain the freshest fruit possible. Before testing, the sample homogeneity was ensured by placing the samples in large, sterile ziplock bags and 
mixing gently before being segregated. The microorganisms present on tomatoes being naturally-occurring microflora might be distributed heterogeneously from fruit to fruit. This allowed even distribution of microbial load or species composition of the initial inoculum. Even though there were chances for variation in species composition among the samples due to factors such as seasonal variation, a region of production, variety of produce, etc., the microflora of these samples were expected to remain fairly similar, with the largest uncontrollable variable being the overall microbial load.

\section{Sample treatment}

Eight quarts of tomatoes (about $4 \mathrm{~kg}$ ) were procured and the worst $5 \%$ that was discolored, physically damaged, or visually molded were discarded. The selected ones were rinsed in distilled water, dried properly, and transferred to sterile ziplock bags and mixed slowly to evenly distribute any initial microbes present on the fruit. Four quarts of tomatoes were retained as a control experiment set, while the other four quarts were coated thrice with $5 \%$ phlorotannin solution on drying. Out of the four quarts of control tomatoes, two quarts were placed in an open sterile plastic tray for observations of the nonchilled treatment, and the other two quarts were placed in sterile zip lock bags and stored in the refrigerator for the chilled treatment. The same was repeated for the treated sets of tomatoes also. Out of the two quarts, quart was for weight loss calculations and the documentation of color, turgor, and overall organoleptic quality. The remaining quart was used for microbial counts.

\section{Sampling process}

From test day 1 , in every third day that followed, for 20 days, the samples were examined visually for organoleptic qualities like brown spots, soft spots, wilting/loss of turgor, visible indicators of microbial growth, etc. and weight was noted. The photos were taken for visual documentation of the sample condition. This data was subsequently correlated with the microbial data obtained. Once the organoleptic studies were done, the samples were returned to their corresponding test environment and the samples for microbial enumeration were prepared.

\section{Plating process}

The plating of the bacterial load on tomato samples was done by the swab/ streak method, and spread plate method. All the required glassware and medium components were autoclaved at $121^{\circ} \mathrm{C}$ at $103.42 \mathrm{kPa}$ for $120 \mathrm{~min}$ to sterilize. All the techniques were done aseptically.

\section{Swab/Streak method}

Bacterial samples were taken from the tomato surface using preautoclaved, sterile cotton swabs rubbed over randomly chosen sample surface for about 1-2 min and the microbe loaded swab was used to streak the agar plates. Proper care was taken not to cut the agar surface. The aseptically prepared plates were incubated in an inverted position at $37^{\circ} \mathrm{C}$ for $24-48 \mathrm{~h}$ for microbial growth.

\section{Spread plate method}

Ten milligrams of flesh from each set of sample was weighed in sterile conditions and homogenized in $10 \mathrm{~mL}$ sterile peptone water $(0.1 \%)$ using pestle and mortar for $5 \mathrm{~min}$. One milliliter of each sample was serially diluted tenfold in sterile distilled water up to 10-5 dilution, from which $0.1 \mathrm{~mL}$ at $10-4$ dilution was spread over the nutrient agar plate using glass L-rod spreaders. The aseptically prepared plates were incubated at $37^{\circ} \mathrm{C}$ for $24-48 \mathrm{~h}$ for the appearance of colonies. After $48 \mathrm{~h}$ incubation, the bacterial colonies were counted by plate counting method.

\section{Total plate count of bacteria (CFU $\mathrm{mL}^{-1}$ )}

The microbial load in each set of tomato was determined as CFU $\mathrm{mL}^{-1}$ using the formula:

$C F U m L^{-1}=[($ No. of colonies $x$ dilution factor $) /$ volume of inoculum $]$

\section{Antimicrobial activity of phlorotannin}

The antimicrobial activity was evaluated by agar well diffusion method. The microbial colonies isolated from the above plates were carefully transferred aseptically using a loop into the nutrient broth $(50 \mathrm{~mL})$ and incubated overnight at room temperature. The following day, the bacterial inoculum was uniformly spread using an L - rod spreader on to nutrient agar plates and allowed to dry. Then wells were made in the seeded plates with the help of a cork-borer. $50 \mu \mathrm{L}$ distilled water was added to the first well as negative control, while $50 \mu \mathrm{L}$ tetracycline was loaded to the second well as positive control. The phlorotannin was loaded to the remaining two wells in a concentration of $50 \mu \mathrm{L}$ and $100 \mu \mathrm{L}$ respectively. The systems were incubated under aerobic conditions for $24 \mathrm{~h}$ at room temperature. After incubation, the zone of inhibition was measured in millimeters.

Similarly, the antimicrobial activity of phlorotannin against two Gram-positive bacteria (Bacillus cereus, Micrococcus luteus) and three Gram-negative bacteria (Pseudomonas aeruginosa, Klebsiella pneumonia, and Escherichia coli) was evaluated by the same method. The microorganism was activated by inoculating a loopful of the strain in nutrient broth $(50 \mathrm{~mL})$ and incubated overnight at room temperature. Then the bacterial inoculum was uniformly spread using a sterile cotton swab on a sterile Petri dish with Mueller-Hinton agar. Thereafter wells were made in the seeded plates with the help of a cork-borer. $10 \mu \mathrm{L}$ of tetracycline was loaded to the first well as positive control. DMSO (Dimethyl sulfoxide) $(1 \mathrm{~mL})$ was dissolved in $9 \mathrm{~mL}$ distilled water and used as a negative control. Phlorotannin was loaded to the remaining three wells in a concentration of 30,60 , and $90 \mu \mathrm{L}$ respectively. The plates were incubated for 24 hours at room temperature under aerobic conditions. After incubation, the inhibition of bacterial growth was measured in millimeters.

\section{Results}

The three seaweeds $S$. myriocystum, T. ornata, and P. tetrastromatica were extracted thrice with $80 \%$ methanol to obtain the crude extracts (Figure 1). The total phenolic content, and the antioxidant activity of the extracts, were evaluated, and the Padina extract with the highest content and activity was further processed. The crude extract was partitioned thrice with equal volumes of dichloromethane and the aqueous phase (AP) was collected and concentrated which was subjected to silica gel column chromatography to obtain two water fractions (WF I \& WF II) and three methanol fractions (MF I, MF II \& MF III) (Figure 1). The total phenolic content, as well as the antioxidant activity of these fractions, was also evaluated. The WF I which had the maximum content and activity was dialyzed and lyophilized to yield the phlorotannin which was further used to treat tomatoes to elucidate the antimicrobial and vegetable spoilage prevention potentials. 


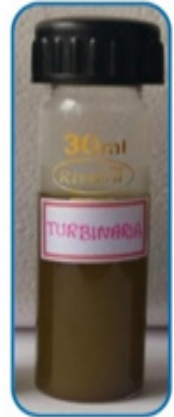

A

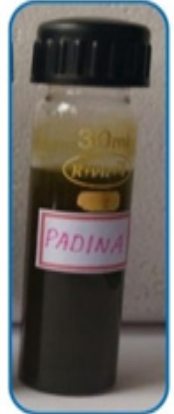

B
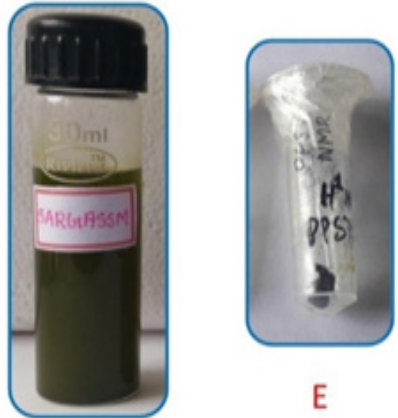

$E$

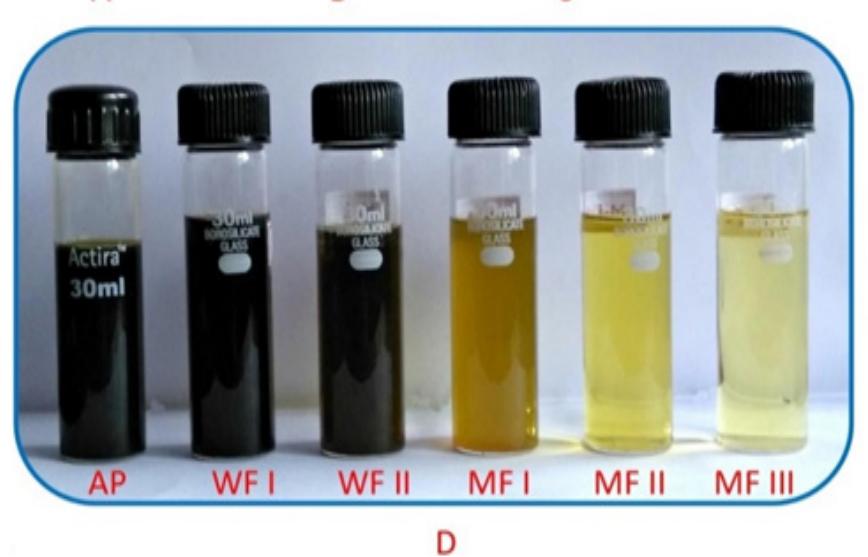

Figure I Algal extracts and fractions: A)Turbinariacrude extract, B) Padinacrude extract, C) Sargassumcrude extract, D) Fractions of Padinacrude extract on silica gel column chromatography:AP, aqueous phase after partitioning with dichloromethane; WF, water fraction; MF, methanol fraction, E) Lyophilized phlorotannin.

\section{Total phenolic content (TPC)}

The highest phenolic content was found in Padina extract (GAE $=$ $\left.0.114 \pm 0.0005 \mathrm{~g} \mathrm{~kg}^{-1}\right)$ followed by Turbinaria $(\mathrm{GAE}=0.097 \pm 0.0005$ $\left.\mathrm{g} \mathrm{kg}^{-1}\right)$ and Sargassum $\left(\mathrm{GAE}=0.075 \pm 0.000 \mathrm{~g} \mathrm{~kg}^{-1}\right)$ extracts. The column fractions of Padina extract exhibited a decreasing order in the total phenolic content, the water fraction I (WF I) having the highest $\left(\mathrm{GAE}=0.026 \pm 0.000 \mathrm{~g} \mathrm{~kg}^{-1}\right)$ (Figure 2).

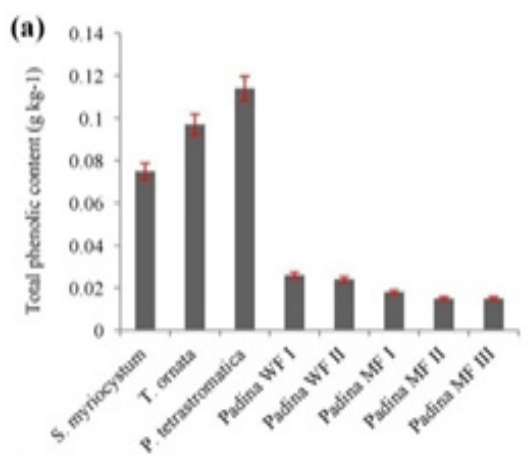

(c)

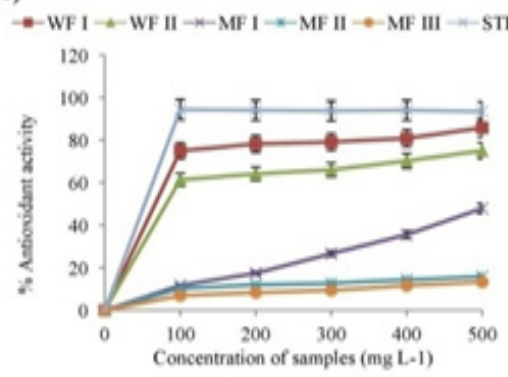

(b)

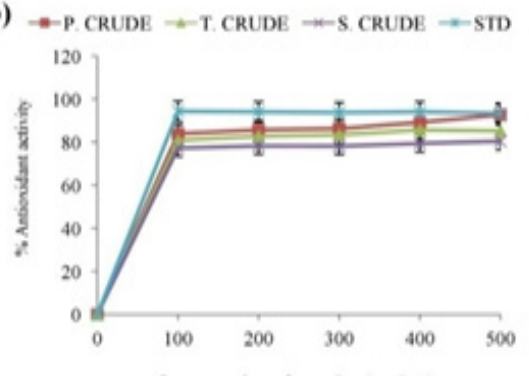

(d)

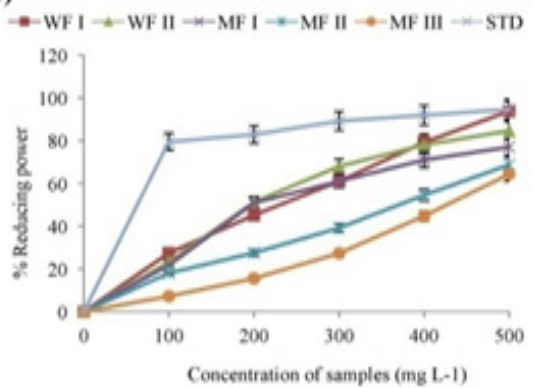

Figure 2 (a). Total phenolic content (TPC) in $\mathrm{g} \mathrm{kg}^{-1}$ Gallic acid Equivalents of the crude extracts of Sargassum, Turbinaria, Padina, water fractions, and methanol fractions of Padina crude extract; (b). Percentage DPPH radical scavenging activity of crude extracts and standard ascorbic acid (STD); (c). Percentage DPPH radical scavenging activity of water fractions, methanol fractions, and standard ascorbic acid (STD); (d). Percentage reducing the power of water fractions, methanol fractions, and standard ascorbic acid (STD). (Mean values $\pm S D ; n=3$ ). 


\section{Antioxidant activity of crude extracts and fractions \\ DPPH radical scavenging activity}

The Figure 2 illustrates the percentage DPPH radical scavenging potential of the various concentrations $(100-500 \mu \mathrm{L})$ of crude methanolic extracts of the three algae and the water fractions and methanol fractions of Padina. The antioxidant activity of the standard ascorbic acid has been given for reference. The highest activity was exhibited by $500 \mu \mathrm{L}$ crude Padina extract $(92.65 \pm 3.43 \%)$ while the least was in $100 \mu \mathrm{L}$ crude Sargassum extract $(77.17 \pm 1.24 \%)$. In case of Padina fractions, the activity was the highest in $500 \mu \mathrm{L}$ of water fraction I (WF I) $(85.76 \pm 3.99 \%)$, and the least in $100 \mu \mathrm{L}$ of methanol fraction III (MF III) $(6.88 \pm 2.56 \%)$. The average activity of the standard ascorbic acid was $93.87 \pm 0.35 \%$.

\section{Reducing power assay}

The reducing power assay was conducted with the fractions of crude Padina extract. The highest activity was observed in the $500 \mu \mathrm{L}$ of water fraction I (WF I) $(93.81 \pm 26.39 \%)$, while the least was in $100 \mu \mathrm{L}$ of methanol fraction III (MF III) $(7.21 \pm 22.99 \%)$. Overall the reducing power of each fraction showed an increase with the increase in concentration, and the same pattern was observed in case of fractions as a whole, i.e., the activity of WF I $>$ WF II $>$ MF I $>$ MF II $>$ MF III (Figure 2).

\section{Fourier transform infra-red (FT-IR) spectroscopy}

The water fraction I (WF I) was characterized using Perkin Elmer System One FTIR. The FT-IR spectral study is preferred because of its non-destructive method, easiness to perform and the meager quantity requirement of the sample. The FT-IR spectrum (Figure 3) showed the following significant wavelengths corresponding to phlorotannins, previously reported. The strong band at $3405 \mathrm{~cm}-1$ denotes the stretching vibrations of O-H groups. The signal at $2924 \mathrm{~cm}-$ 1 corresponds to the $\mathrm{C}-\mathrm{H}$ stretch vibration. The band at $662 \mathrm{~cm}^{-1}$ was attributed to the stretching vibration of the benzene ring containing aromatic substitution. ${ }^{22}$ The band at $1453 \mathrm{~cm}^{-1}$ corresponds to the aromatic nuclei. The band at $1037 \mathrm{~cm}^{-1}$ corresponds to the glycosidic linkage vibrations of $\mathrm{C}-\mathrm{O}-\mathrm{C}$ and $\mathrm{C}-\mathrm{O}-\mathrm{H}$, indicating the presence of some carbohydrates in the sample. ${ }^{23}$

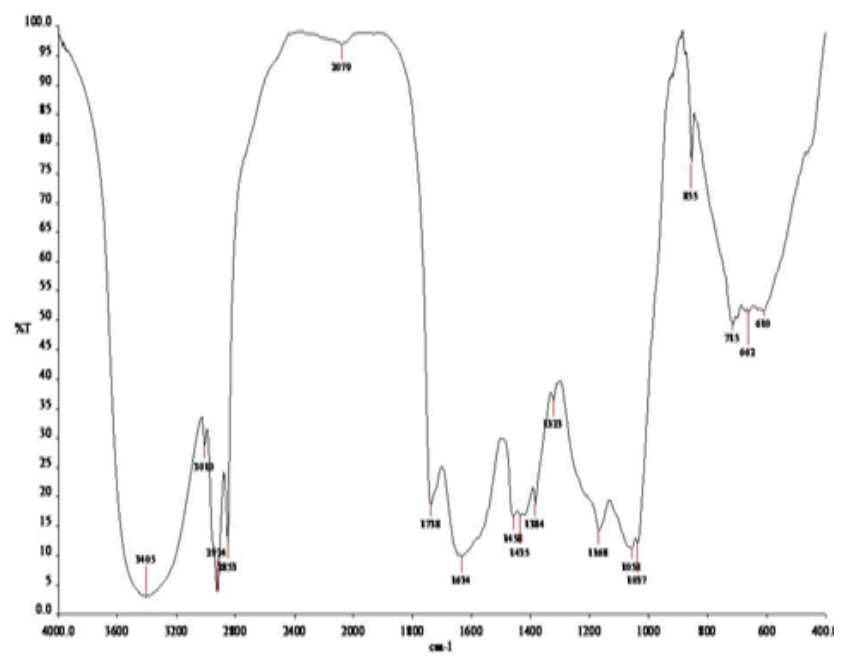

Figure 3 FT-IR spectrum of phlorotannin from Padina tetrastromatica.

\section{Assessment of antimicrobial activity and spoilage prevention potential of phlorotannin (WF I) on tomatoes}

The role of antioxidant and antimicrobial properties of phlorotannin in spoilage prevention and shelf life enhancement in an easily perishable vegetable like tomato was assessed. Figure 4 shows the various stages in the ripening and spoilage of tomatoes, and the treated and control set of tomatoes. The organoleptic properties recorded are given in Table 1.

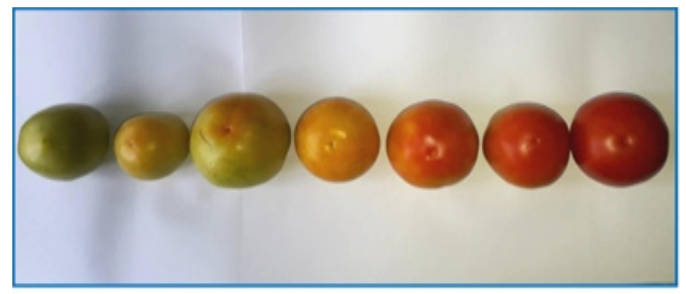

A

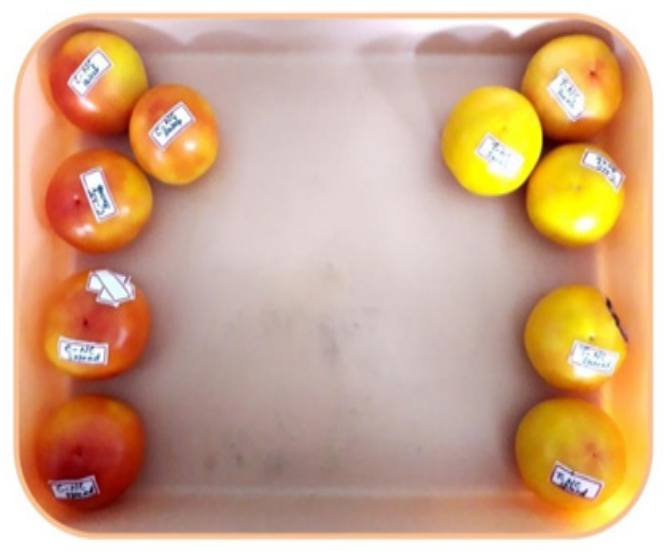

B

Figure $4 \mathrm{~A}$ - Various stages in the ripening and spoilage of tomatoes; $\mathrm{B}$ - The control set of tomatoes (left) and the treated set of tomatoes (right).

From the table, it is clear that the spoilage and the change in all parameters were initiated on 6th observation day after treatment, gradually leading to a slow decay process. Visible indicators of microbial spoilage were absent during the study period, but the presence and extent of microbial load on samples were elucidated using plating techniques. One important observation was that the color of treated tomatoes turned yellow from green but remained yellow throughout the study. Whereas the untreated set of tomatoes tuned red quicker. There was a slight weight loss from the third observation day, and it gradually increased with time. The microbial load on unwashed, untreated tomatoes was the highest. The treated tomatoes exhibited a higher microbial load than the control at the beginning of the experiments. This may be due to the dampness of the phlorotannin solution used to treat the tomatoes. On the second observation day, swab plates of treated batch showed microbial growth than the control plate, and vice versa in spread plates. The trend of microbial growth reversed on the third observation day when the control batch of both chilled and non-chilled set exhibited a higher microbial load than the treated batch of the chilled and non-chilled set. On the fourth observation day also, the microbial growth was higher in control chilled and non-chilled batches than in treated batches of tomatoes. There was a gradual increase in the microbial load in all sets of samples, but lesser in treated sets. The chilled sets of tomatoes 
showed early color changes and an indication of spoilage than the non-chilled sets stored in open lab space. The high antioxidant and antimicrobial properties of phlorotannins might be preventing the color change and early spoilage in tomatoes. The treated tomatoes retained fresh appearance as compared to their untreated counterparts. There were four colonies observed in the plates: white rhizoid raised colony, reddish circular flat colony, pale white circular flat colony, and a yellow circular flat colony. The red, white, and pale white colonies were Gram-negative, whereas the yellow colony was Gram-positive. The colony-forming units (CFU) or cells per milliliter of the samples on each observation day was calculated using the formula (Table 2). The CFU mL $\mathrm{mL}^{-1}$ range from 106 to 108 cells $\mathrm{mL}^{-1}$ indicates spoilage.

Table I Organoleptic/ sensory indicators observed in control and treated tomatoes on every third day after treatment, for twenty days. ( $x$ ) Indicates absence $\&(\square)$ indicates the presence of a property. CC, control chilled; CNC, control non-chilled;TC, treated chilled;TNC, treated non-chilled

\begin{tabular}{|c|c|c|c|c|c|c|c|c|c|c|}
\hline $\begin{array}{l}\text { Observation } \\
\text { days }\end{array}$ & $\begin{array}{l}\text { Experiment } \\
\text { sets }\end{array}$ & $\begin{array}{l}\text { Soft } \\
\text { spots }\end{array}$ & $\begin{array}{l}\text { Brown } \\
\text { Spots }\end{array}$ & $\begin{array}{l}\text { Color } \\
\text { changes }\end{array}$ & $\begin{array}{l}\text { Loss of } \\
\text { turgor }\end{array}$ & Texture/ & Odors & Visible & Weight & $\begin{array}{l}\text { Weightloss } \\
\text { (\%) }\end{array}$ \\
\hline & & & & & & Hardness & & $\begin{array}{l}\text { Indicatc } \\
\text { microbi }\end{array}$ & $\begin{array}{l}\text { spoilage } \\
\text { spoing }\end{array}$ & \\
\hline \multirow[t]{4}{*}{ Ist day } & $\mathrm{CC}$ & $x$ & $x$ & $x$ & $x$ & $x$ & $\checkmark$ & $x$ & 174.94 & 0 \\
\hline & CNC & $x$ & $x$ & $x$ & $x$ & $x$ & $\checkmark$ & $x$ & 275.39 & 0 \\
\hline & $\mathrm{TC}$ & $x$ & $x$ & $x$ & $x$ & $x$ & $\checkmark$ & $x$ & 183.06 & 0 \\
\hline & TNC & $x$ & $x$ & $x$ & $x$ & $x$ & $\checkmark$ & $x$ & 209.52 & 0 \\
\hline \multirow[t]{4}{*}{ 2nd day } & $\mathrm{CC}$ & $x$ & $x$ & $x$ & $x$ & $x$ & $\checkmark$ & $x$ & I74.94 & 0 \\
\hline & $\mathrm{CNC}$ & $x$ & $x$ & $x$ & $x$ & $x$ & $\checkmark$ & $x$ & 275.39 & 0 \\
\hline & $\mathrm{TC}$ & $x$ & $x$ & $x$ & $x$ & $x$ & $\checkmark$ & $x$ & 183.06 & 0 \\
\hline & TNC & $x$ & $x$ & $x$ & $x$ & $x$ & $\checkmark$ & $x$ & 209.52 & 0 \\
\hline \multirow[t]{4}{*}{$3 r d$ day } & $\mathrm{CC}$ & $x$ & $x$ & $\checkmark$ & $\checkmark$ & $\checkmark$ & $\checkmark$ & $x$ & 174.62 & 5 \\
\hline & $\mathrm{CNC}$ & $x$ & $x$ & $\checkmark$ & $\checkmark$ & $\checkmark$ & $\checkmark$ & $x$ & 274.27 & 9 \\
\hline & $\mathrm{TC}$ & $x$ & $x$ & $\checkmark$ & $x$ & $x$ & $x$ & $x$ & 183.01 & 6 \\
\hline & TNC & $x$ & $x$ & $\checkmark$ & $x$ & $x$ & $x$ & $x$ & 206.48 & 8 \\
\hline \multirow[t]{4}{*}{ 4th day } & $\mathrm{CC}$ & $x$ & $x$ & same & $\checkmark$ & same & $\checkmark$ & $x$ & 173.58 & 9 \\
\hline & $\mathrm{CNC}$ & $x$ & $x$ & same & $\checkmark$ & same & $\checkmark$ & $x$ & 274.12 & 2 \\
\hline & $\mathrm{TC}$ & $x$ & $x$ & same & $x$ & same & $\checkmark$ & $x$ & 182.98 & 8 \\
\hline & TNC & $x$ & $x$ & same & $x$ & same & $\checkmark$ & $x$ & 204.51 & 9 \\
\hline \multirow[t]{4}{*}{ 5th day } & $\mathrm{CC}$ & $x$ & $x$ & same & same & same & same & $x$ & 173.02 & I \\
\hline & $\mathrm{CNC}$ & $x$ & $x$ & same & same & same & same & $x$ & 273.99 & 6 \\
\hline & $\mathrm{TC}$ & $x$ & $x$ & same & same & same & same & $x$ & 182.95 & 6 \\
\hline & TNC & $x$ & $x$ & same & same & same & same & $x$ & 204.48 & I \\
\hline \multirow[t]{4}{*}{ 6th day } & $\mathrm{CC}$ & $x$ & $\checkmark$ & $\checkmark$ & same & $\checkmark$ & $\checkmark$ & $x$ & 172.96 & I \\
\hline & $\mathrm{CNC}$ & $x$ & $\checkmark$ & $\checkmark$ & same & $\checkmark$ & $\checkmark$ & $x$ & 273.92 & 8 \\
\hline & $\mathrm{TC}$ & $x$ & $\checkmark$ & $\checkmark$ & same & $\checkmark$ & $\checkmark$ & $x$ & 182.89 & 6 \\
\hline & TNC & $x$ & $\checkmark$ & $\checkmark$ & same & $\checkmark$ & $\checkmark$ & $x$ & 204.32 & 6 \\
\hline \multirow[t]{4}{*}{ 7th day } & $\mathrm{CC}$ & $x$ & same & same & $x$ & same & $\checkmark$ & $x$ & 172.73 & 9 \\
\hline & $\mathrm{CNC}$ & $x$ & same & same & $x$ & same & $\checkmark$ & $x$ & 273.58 & 4 \\
\hline & $\mathrm{TC}$ & $x$ & same & same & $x$ & same & $\checkmark$ & $x$ & 182.74 & 6 \\
\hline & TNC & $x$ & same & same & $x$ & same & $\checkmark$ & $x$ & 204.23 & I \\
\hline \multirow[t]{4}{*}{ 8th day } & $\mathrm{CC}$ & $x$ & $\checkmark$ & $\checkmark$ & $\checkmark$ & same & $\checkmark$ & $x$ & 172.57 & 5 \\
\hline & CNC & $x$ & $\checkmark$ & $\checkmark$ & $\checkmark$ & same & $\checkmark$ & $x$ & 273.25 & 6 \\
\hline & $\mathrm{TC}$ & $x$ & $\checkmark$ & $\checkmark$ & $\checkmark$ & same & $\checkmark$ & $x$ & 182.82 & 6 \\
\hline & TNC & $x$ & $\checkmark$ & $\checkmark$ & $\checkmark$ & same & $\checkmark$ & $x$ & 204.12 & I \\
\hline
\end{tabular}


Table 2 Colony-forming unit (CFU mL-1) of the samples at 10-4 dilution on Ist, 2 nd, 3 rd, and 4 th observation days

\begin{tabular}{lcccc}
\hline & \multicolumn{4}{c}{ Colony forming unit $($ CFU mL-1) } \\
\cline { 2 - 5 } Type of Plates & Day I & Day 2 & Day 3 & Day 4 \\
\hline Control Chilled & $5.68 \times 10^{5}$ & $5.94 \times 10^{5}$ & $4.26 \times 10^{6}$ & $4.63 \times 10^{6}$ \\
Treated Chilled & $4.20 \times 10^{5}$ & $4.82 \times 10^{5}$ & $3.09 \times 10^{6}$ & $3.29 \times 10^{6}$ \\
Control & $6.12 \times 10^{5}$ & $6.54 \times 10^{5}$ & $5.17 \times 10^{6}$ & $5.37 \times 10^{6}$ \\
Non-Chilled & & & & \\
Treated & $5.63 \times 10^{5}$ & $5.81 \times 10^{5}$ & $3.92 \times 10^{6}$ & $4.16 \times 10^{6}$ \\
Non-Chilled & & & & \\
\hline
\end{tabular}

\section{Antimicrobial activity of phlorotannin}

The microbial colonies isolated from the plates were developed into broth cultures and the phlorotannin was directly tested for its antimicrobial activity using agar well diffusion assay. The zone of inhibition of various microbes has been given in Table 3 . The highest antimicrobial activity (39 $\mathrm{mm}$ diameter) was observed in $100 \mu \mathrm{L}$ of WF I against the red colony, while the least activity $(1.3$ $\mathrm{mm}$ diameter) was observed in the $100 \mu \mathrm{L}$ of WF I against yellow colony. In accordance with these values, it was observed in the agar plates that in course of time, the red colonies had disappeared in the treated tomatoes, while the white and yellow colonies were present but in lesser amounts than in the control batch. These findings hence point towards the efficient antimicrobial activity of phlorotannins in the prevention of early spoilage in tomatoes. The inhibition of fungal components was however not studied. The phlorotannin also exhibited antimicrobial activity at higher concentrations against all the five microbes tested (Table 4).

Table 3 Zone of inhibition (including $8 \mathrm{~mm}$ borer diameter) of various concentrations of Padina phlorotannin against vegetable spoilage microbes in tomato (Note: PC,positive control; NC, negative control)

\begin{tabular}{llll}
$\begin{array}{l}\text { Conc. of } \\
\text { PadinaPhlorotannin }\end{array}$ & Zone of Inhibition $(\mathbf{m m})$ & \\
\hline & Microbe A & Microbe B & Microbe C \\
\hline & (red) & (yellow) & (white) \\
\hline PC & 44 & 2.6 & 3.7 \\
NC & - & - & - \\
30 & 33 & - & 1.7 \\
50 & 27 & - & 2.5 \\
70 & 36 & - & 3.1 \\
\hline 100 & 39 & I.3 & \\
\hline
\end{tabular}

Table 4 Zone of inhibition (including $8 \mathrm{~mm}$ borer diameter) of various concentrations of Padinaphlorotannin against selected food borne microbes (Note: PC, positive control; NC, negative control)

\begin{tabular}{llllll}
\hline \multicolumn{5}{c}{$\begin{array}{l}\text { Padina tetrastromatica } \\
\text { phlorotannin }\end{array}$} \\
\hline Algal extract & $\begin{array}{l}\text { (Zone of } \\
\text { inhibition(mm)) }\end{array}$ & & \\
\hline Concentration $(\mu \mathrm{L})$ & PC & NC & 30 & 60 & 90 \\
Escherichia coli & 16 & - & - & 12 & 13 \\
Klebsiella pneumoniae & 15 & - & - & 10 & 12 \\
Pseudomonas aeruginosa & 18 & - & II & 14 & 16 \\
Micrococcus luteus & 13 & - & II & 13 & 13 \\
Bacillus cereus & 30 & - & 12 & 15 & 17 \\
\hline
\end{tabular}

\section{Discussion}

In brown algae, the total phenolic content estimation corresponds to the content of phlorotannin in them, as phlorotannins form their main phenolic constituent. Phlorotannins are highly hydrophilic and exist abundantly in marine brown algae as an integral component of their cell wall as well as in vesicles called physodes. ${ }^{24}$ The phlorotannins stored in physodes are soluble, while that of cell walls are insoluble, forming complexes with proteins and alginic acid. ${ }^{23}$ In previous studies, the phlorotannin levels reported were nearly $20 \%$ in Fucales and 30\% in Dictyotales (dry weight). The content of cell-wall bound phlorotannins is usually much lower than the amount of soluble/ free phlorotannins. ${ }^{25}$ The amounts found in this study was $0.15,0.48$, $0.57,0.13,0.12,0.09,0.075$, and $0.075 \%$ in Sargassum, Turbinaria, Padina, WF I, WF II, MF I, MF II and MF III respectively which is considerably lower (i.e., $\leq 0.1 \%$ of dry matter). This difference in yield may be attributed to the factors like species, geographic location of collection site, period collection, environmental factors, and the extraction and purification techniques employed. Also, phlorotannins being hydrophilic and polar are more soluble in polar solvents like water, methanol, ethanol, etc., and a multi-component solvent system like $80 \%$ methanol effectively extracts out phlorotannins.

The antioxidant activity observed in crude Padina extract was comparable to that shown by the ascorbic acid, which further supports the findings previously published. The overall activity of water fractions was higher compared to the methanol fractions, due to higher amounts of phlorotannins in water fractions (especially WF I) as the polarity of water is more than methanol. The antioxidant property of the phlorotannins obtained from brown seaweeds has already been reported worldwide. Free radical scavenging property is crucial for any drug candidate molecule or food industry targeted molecule for imparting its benefits effectively. The antioxidant property of phlorotannins has been reported to be comparable with that of many commercially available antioxidant compounds like ascorbic acid and $\alpha$-tocopherol. ${ }^{26}$ It has been reported that the aqueous extract of Padina tetrastromatica and Padina gymnospora from Mandapam coast in Tamil Nadu, showed higher TPC which corresponded with higher DPPH scavenging of the extracts. The present findings correlated with this report. It was also noted that polar solvent extracts of brown algae exhibited high antioxidant properties, and the chemical composition analysis of these extracts revealed the presence of radical scavengers such as phlorotannins and fucoxanthin. ${ }^{27}$ The high activity observed for Padina may be due to the abundance of 
low molecular weight phlorotannin polymers, which are known to be powerful antioxidants. The low degree of polymerization allows more number of free hydroxyl units which in turn allows a higher level of hydrogen donation and consequent stabilization of free radicals. ${ }^{28}$ Phlorotannins purified from the brown algae such as E. bicyclis, E. kurome, H. fusiformis, U. pinnatifida, and E. cavahave displayed potent antioxidant effects comparable to antioxidants like ascorbic acid and $\alpha$-tocopherol as reported earlier. ${ }^{15}$

The antimicrobial study of algal phenolics has been reported specifically against Staphylococcus aureus and Bacillus spp. The methanol and diethyl ether extracts of brown seaweeds like Padina gymnospora, Turbinariaornata, Turbinariaconoides, Dictyotadichotoma, Ascophyllum nodosum, and Ecklonia cava, containing phlorotannins were usually reported with higher antimicrobial activity against a wide range of bacterial pathogens like Staphylococcus aureus, Escherichia coli, Salmonella typhimurium, Bacillus cereus, Listeria monocytogenes etc. ${ }^{4}$

The use of seaweed extracts to deal with the postharvest loss of fruit started around the 2000s. A commercial seaweed extract (Cytolan Star ${ }^{\circledR}$ ) was found to improve the quality of orange fruit (cv. Valencia) during storage and maintained for a longer period. Thereafter the use of seaweed extracts for preventing microbial spoilage and pathogenic attack on crops was investigated further The ethanolic extract of the red alga Solieriarobusta was found to inhibit five fruit spoiling fungi Aspergillus flavus, A. niger, A. ochraceus, P. funiculosum, and Phytophthora infestans, and also the growth of Macrophominaphaseolina, R. solani, and Fusarium solani. Besides, the seaweed extracts also stimulated growth in strawberry by preventing pathogenic attacks and physiological hazards both in vivo and under storage. ${ }^{21}$

\section{Conclusion}

The three brown algae were found to be valuable sources of phlorotannins, reported with a wide range of biological activities. In this study, $P$. tetrastromatica exhibiting the highest total phenolic content showed effective antimicrobial and antioxidant potential against food spoilage microbes. The promising results of bacterial growth inhibition and inactivation by purified Padina phlorotannin and the lack of studies carried out in real foods open a novel research niche with a high application at food industrial level. This is the first report on the direct application of phlorotannin from $P$. tetrastromatica on tomatoes for spoilage prevention. The algal product requires further elaborate exploration of its stability and properties for its direct incorporation in food or use as a natural food preservative. Consequently, the twofold use of algal bioactive as an antimicrobial and antioxidant in food products has good prospects in the preservation of easily perishable vegetables. It helps in the development and innovation of sustainable food products that are as natural as possible. Also, the phlorotannins being water-soluble, its coating can be easily removed before consumption, unlike the other preservatives that require chemicals or specific solutions for their removal. Hence this suggests that further research may give insights into the proper utilization of macroalgae like $P$. tetrastromatica for the largescale extraction of a natural and powerful antioxidant and antimicrobial agent like phlorotannin for application in foods, both as a preservative and a nutraceutical.

\section{Acknowledgments}

The authors greatly acknowledge and thank The Director, Center for Advanced Studies in Botany, University of Madras, Guindy Campus, Chennai, India for providing laboratory facilities. We are also thankful to the Sophisticated Analytical Instrumentation Facility, Indian Institute of Technology, Madras, India for providing the analytical facilities for carrying out this research.

\section{Conflicts of interest}

The authors declare that there was no conflict of interest.

\section{Funding}

None.

\section{References}

1. Leff JW, Fierer Noah. Bacterial communities associated with the surfaces of fresh fruits and vegetables. PloS One. 2013;8(3):e59310.

2. Kaur P, Rai N. Bacteriological analysis of fresh vegetables from main market of Dehradun. Intl Journal of PharmTech Res. 2015;8(3):415-425.

3. Rawat Seema. Food Spoilage: Microorganisms and their prevention. Asian Journal of Plant Sci and Res. 2015;5(4):47-56.

4. Pina-Pérez MC, Rivas A, Martínez A, et al. Antimicrobial potential of macro and microalgae against pathogenic and spoilage microorganisms in food. Food Chemistry. 2017;235:34-44.

5. Jégou Camille, Kervarec Nelly, Cérantola Stéphane, et al. NMR use to quantify phlorotannins: The case of Cystoseira tamariscifolia, a phloroglucinol-producing brown macroalga in Brittany (France). Talanta. 2015;135:1-6.

6. Li Yajing, Fu Xiaoting, Duan Delin, et al. Extraction and identification of phlorotannins from the brown alga, Sargassum fusiforme (Harvey) Setchell. Marine Drugs. 2017;15(2):49.

7. Kim Jiyoung, Yoon Minseok, Yang Hyejin, et al. Enrichment and purification of marine polyphenol phlorotannins using macroporous adsorption resins. Food chemistry. 2014;162:135-142.

8. Mwangi Henry M, Van Der Westhuizen, Jan Marnewick, et al. Isolation, identification and radical scavenging activity of phlorotannin derivatives from brown algae, Ecklonia maxima: An experimental and theoretical study. Free Radicals and Antioxidants. 2013;3:S1-S10.

9. Leyton A, Vergara-Salinas JR, Pérez-Correa JR, et al. Purification of phlorotannins from Macrocystis pyrifera using macroporous resins. Food Chemistry. 2017;237:312-319.

10. Balboa Elena M, Conde Enma, Moure Andres, et al. In vitro antioxidant properties of crude extracts and compounds from brown algae. Food Chemistry. 2013;138(2-3):1764-1785.

11. Sanjeewa Kalu Kapuge Asanka, Kim Eun-A, Son Kwang-Tae, et al. Bioactive properties and potentials cosmeceutical applications of phlorotannins isolated from brown seaweeds: A review. Journal of Photochemistry and Photobiology B: Biology. 2016;162:100-105.

12. Wang Hui-Min David, Li Xiao-Chun, Lee Duu-Jon, et al. Potential biomedical applications of marine algae. Bioresource Technology. 2017;244:1407-1415.

13. Eom Sung-Hwan, Kim Young-Mog, Kim Se-Kwon. Antimicrobial effect of phlorotannins from marine brown algae. Food and Chemical Toxicology. 2012;50(9):3251-3255.

14. Gyawali R, Ibrahim SA. Natural products as antimicrobial agents. Food Control. 2014;46:412-429.

15. Suleria Hafiz Ansar Rasul, Gobe Glenda, Masci Paul, et al. Marine bioactive compounds and health promoting perspectives; innovation pathways for drug discovery. Trends in Food Sci \& Tech. 2016;50:44-55.

16. Rout Sradhasini, Kumar Anjan. A review on the potentiality of marine seaweeds as a medicinal source. World Journal of Pharmacy and Pharmaceutical Sciences. 2015;4(10):458-476. 
17. de Sousa Carolina Bruno, Gangadhar Katkam N, Macridachis Jorge, et al. Cystoseira algae (Fucaceae): Update on their chemical entities and biological activities. Tetrahedron: Asymmetry. 2017;28:1486-1505.

18. Singleton Vernon L, Orthofer Rudolf, Lamuela-Raventós Rosa M. Analysis of total phenols and other oxidation substrates and antioxidants by means of folin-ciocalteu reagent. Methods in Enzymology. 1999;299:152-178).

19. Vani T, Rajani M, Sarkar S, et al. Antioxidant properties of the ayurvedic formulation Triphala and its constituents. Int. Journal of Pharmacognosy. 1997;35(5):313-337.

20. Oyaizu M. Studies on products of browning reactions: Antioxidant activities of products of browning reaction prepared from glucosamine. J Nutrit. 1986;44:307-315.

21. de Corato Ugo, Salimbeni Rocco, De Pretis Agostino, et al. Antifungal activity of crude extracts from brown and red seaweeds by a supercritical carbon dioxide technique against fruit postharvest fungal diseases. Postharvest Biology and Technology. 2017;13:16-30.

22. Sellimi Sabrine, Benslima Abdelkarim, Barragan-Montero Veronique, et al. Polyphenolic-protein-polysaccharide ternary conjugates from Cystoseira barbata Tunisian seaweed as potential biopreservatives: Chemical, antioxidant and antimicrobial properties. Int Journal of Biol. Macromolecules. 2017;105:1375-1383.
23. Leyton A, Pezoa-Conte Ricardo, Barriga A, et al. Identification and efficient extraction method of phlorotannins from the brown seaweed Macrocystis pyrifera using an orthogonal experimental design. Algal Research. 2016;16:201-208.

24. Alassali Ayah, Cybulska Iwona, Brudecki Grzegorz Przemyslaw, et al. Methods for upstream extraction and chemical characterization of secondary metabolites from algae biomass. Adv Tech in Biol \& Med. 2016;4(1):1-16.

25. Ferreres Federico, Lopes Graciliana, Gil-Izquierdo Angel, et al Phlorotannin extracts from Fucales characterized by HPLC-DAD-ESIMSn: Approaches to hyaluronidase inhibitory capacity and antioxidant properties. Marine Drugs. 2012;10(12):2766-2781.

26. Sathya R, Kanaga N, Sankar P, et al. Antioxidant properties of phlorotannins from brown seaweed Cystoseira trinodis (Forsskål) C. Agardh. Arabian Journal of Chemistry. 2017;10:S2608-S2614.

27. Chakraborty Kajal, Praveen Nammunayathuputhenkotta Krishnankartha, Vijayan Kodayan Kizekadath, et al. Evaluation of phenolic contents and antioxidant activities of brown seaweeds belonging to Turbinaria spp. (Phaeophyta, Sargassaceae) collected from Gulf of Mannar. Asian Pacific Journal of Tropical Biomedicine. 2013;3(1):8-16.

28. Kirke DA, Smyth TJ, Rai DK, et al. The chemical and antioxidant stability of isolated low molecular weight phlorotannins. Food Chemistry. 2017;221:1104-1112. 